\title{
Involvement of nuclear factor I-A1 in the regulation of regucalcin gene promoter activity in cloned normal rat kidney proximal tubular epithelial cells
}

\author{
NATSUMI SAWADA and MASAYOSHI YAMAGUCHI \\ Laboratory of Endocrinology and Molecular Metabolism, Graduate School of Nutritional Sciences, \\ University of Shizuoka, 52-1 Yada, Suruga-ku, Shizuoka 422-8526, Japan
}

Received May 26, 2006; Accepted July 19, 2006

\begin{abstract}
Nuclear factor I-A1 (NF1-A1) can bind to the TTGGC motif in the rat regucalcin gene promoter region. This study was undertaken to determine whether NF1-A1 is involved in the enhancement of the rat regucalcin gene promoter activity using the $-710 /+18$ LUC construct (wildtype) or $-710 /+18$ LUC construct with the deletion of $-523 /-435$ including the TTGGC motif (mutant) in cloned normal rat kidney proximal tubular epithelial NRK52E cells. Cells were transfected with the $-710 /+18$ LUC construct vector or the $-710 /+18$ LUC construct with the deletion of $-523 /-435$. NRK52E cells (wild-type) or NRK52E cells transiently transfected with HA-NF1-A1/phCMV2 were cultured for $48 \mathrm{~h}$ in a medium containing either vehicle or BS $(5 \%)$ in the presence or absence of various factors. HA-NF1-A1 was localized in the nucleus of wild-type cells. Luciferase activity was significantly increased as compared to that of wild-type cells. This increase was significantly enhanced in the presence of phorbol 12-myristate 13-acetate (PMA; $10^{-6} \mathrm{M}$ ). Such an enhancement was not seen by culture with Bay K $8644\left(10^{-6} \mathrm{M}\right)$ or dibutyryl cyclic AMP $\left(10^{-4} \mathrm{M}\right)$. The increase in luciferase activity in NRK52E cells transfected with HANF1-A1 was not observed in the presence of dibucaine $\left(10^{-6}\right.$ $\mathrm{M})$, staurosporine $\left(10^{-9} \mathrm{M}\right)$, or PD $98059\left(10^{-8} \mathrm{M}\right)$, which is an inhibitor of various protein kinases. Such an inhibition was also seen in the presence of vanadate $\left(10^{-6} \mathrm{M}\right)$ or okadaic acid $\left(10^{-6} \mathrm{M}\right)$, an inhibitor of protein phosphatase. The increase in luciferase activity in NRK52E cells transfected with HA-NF1-A1/ phCMV2 was not seen in the mutant with deletion of $-523 /-435$. The increase in luciferase activity in HA-NF1-A1/ phCMV2-transfected NRK52E cells
\end{abstract}

Correspondence to: Dr Masayoshi Yamaguchi, Laboratory of Endocrinology and Molecular Metabolism, Graduate School of Nutritional Sciences, University of Shizuoka, 52-1 Yada, Surugagu, Shizuoka 422-8526, Japan

E-mail: yamaguch@u-shizuoka-ken.ac.jp

Key words: nuclear factor I-A1, RGPR-p117, regucalcin, gene expression, signaling factor was not significantly enhanced in the cells transiently cotransfected with HA-RGPR-p117/phCMV2, which could increase the regucalcin gene promoter activity using the 710/+18 LUC construct (wild-type). This study demonstrates that NF1-A1 enhances the regucalcin promoter activity which is related to the TTGGC motif, and that its enhancing effect is partly mediated through phosphorylation in NRK52E cells.

\section{Introduction}

Regucalcin was discovered as a novel $\mathrm{Ca}^{2+}$-binding protein not including the EF-hand motif, which differs from calmodulin, in the liver cytosol of rats $(1,2)$. The name regucalcin was proposed for this $\mathrm{Ca}^{2+}$-binding protein, that regulates the $\mathrm{Ca}^{2+}$ and/or calmodulin effects on various enzymes in liver cells $(3,4)$. In recent years, regucalcin has been demonstrated to play a multifunctional role as a regulatory protein in the intracellular signaling pathway in many cell types (reviewed in refs. 5-7).

The gene of regucalcin is highly conserved in vertebrate species $(2,8)$. The rat and human regucalcin gene consists of seven exons and six introns, and several consensus regulatory elements exist in the upstream of the 5'-flanking region of the gene (9). The promoter characterization of the 5'-flanking region of the rat regucalcin gene and its transcriptional regulation by various signaling factors has been shown $(10,11)$. We determined the nuclear factor binding site, which contains a nuclear factor I (NF1)-like motif TTGGC(N) 6 CC (12), in the promoter region of the rat regucalcin gene (13). The binding of nuclear protein to the TTGGC sequence of the regucalcin gene has been shown to enhance the phosphorylation of hepatic nuclear protein that is mediated through $\mathrm{Ca}^{2+}$-dependent protein kinases (14). NF1-A1 and RGPRp117 are identified as a hepatic nuclear factor that binds to the TTGGC sequence of the rat regucalcin gene promoter region using a yeast one-hybrid system $(15,16)$.

RGPR-p117, which was discovered as a regucalcin gene promoter region-related protein $(16,17)$, is localized in the nuclei of cloned normal rat kidney proximal tubular epithelial NRK52E cells (18). Overexpression of RGPR-p117 has been shown to enhance the expression of regucalcin mRNA and its protein level in NRK52E cells (19). RGPR-p117 can enhance the rat regucalcin gene promoter activity that is related to the TTGGC motif in NRK52E cells (20). NF1-A1 has been shown 
to bind the TTGGC motif using gel shift assay (8). Whether NF1-A1 regulates the rat regucalcin gene promoter activity is unknown, however.

This study was undertaken to determine whether NF1-A1 enhances the rat regucalcin gene promoter activity in cloned normal rat kidney proximal tubular epithelial NRK52E cells using the method of transfection of HA-NF1-A1/phCMV2. We found that NF1-A1 localizes in the nuclei of NRK52E cells, and that it increases the rat regucalcin gene promoter activity, which involves the TTGGC motif in the cells.

\section{Materials and methods}

Chemicals. Non-essential amino acid solution, bovine serum (BS) and penicillin-streptomycin solution $(5,000 \mathrm{U} / \mathrm{ml}$ penicillin; $5,000 \mu \mathrm{g} / \mathrm{ml}$ streptomycin) were obtained from Gibco Laboratories (Grand Island, NY). Dulbecco's modified Eagle's medium (DMEM), staurosporine, PD98059, dibucaine, Bay K 8644, parathyroid hormone [synthetic human PTH (134)], DcAMP ( ${ }^{6}, 2^{\prime}$-dibutyryl cyclic adenosine $3^{\prime}, 5^{\prime}$ monophosphate), phorbol 12-myristate 13-acetate (PMA), and okadaic acid were obtained from Sigma Chemicals (St. Louis, MO). Vanadate and other chemicals were purchased from Wako Pure Chemical Co. (Osaka, Japan). pGL3-basic vector and pRL-TK vector were purchased from Promega (Madison, WI). The reagents used were dissolved in distilled water or ethanol, and some reagents were passed through an ion exchange resin to remove metal ions.

Cell culture. Cloned normal rat kidney proximal tubular epithelial cells (NRK52E; $1 \times 10^{5}$ cells) $(21,22)$ were maintained in DMEM supplemented with $5 \% \mathrm{BS}$, nonessential amino acids solution, $50 \mathrm{U} / \mathrm{ml}$ penicillin, and $50 \mu \mathrm{g} /$ $\mathrm{ml}$ streptomycin in humidified $5 \% \mathrm{CO}_{2} / 95 \%$ air at $37^{\circ} \mathrm{C}$ to obtain subconfluent monolayers.

Construction of the reporter gene plasmids. The reporter gene plasmids were generated with the subcloning of a restriction fragment from $-710 /+18$ TA vector (13). The DNA fragment $-710 /+18$ of the rat regucalcin gene was prepared from a vector by $\mathrm{KpnI} / \mathrm{XhoI}$ restriction digestion, and then cloned into the pGL3-Basic promoterless plasmid containing the firefly luciferase gene (11). This wild-type promoter construct was named the $-710 /+18$ LUC (WT). The DNA fragment (-710/ -523) was amplified by 2 primers 5 'AGGTACCGAATTCCTGACTGATCTTT-3' and 5'AATGAGCTCCAGGCAAAGAACATTCTAT-3' and digested by EcoRI and SacI, and the fragment was ligated into the EcoRI and SacI site of -710/+18 LUC (WT) and its construct was named Mut 1.

Construction of HA-NF1-Al and HA-RGPR-p117 expression plasmids. For construction of the HA-tagged NF1-A1 expression vector, DNA covering the N-terminal fragment of NF1-A1 was amplified by PCR with two primers 5'-TAGA TCTGTATTCTCCGCTCTGTCTCA-3' and 5'-CTATGTGA TGGGGCTGGACACAGAG-3' using GST-NF1-A1/pGEX$4 \mathrm{~T}$ as a template and digested with $B g l \mathrm{II}$ and $E c o$ RV. The Cterminal fragment of NF1-A1 was obtained by digestion of GST-NF1-A1/pGEX-4T with EcoRV and NotI. These two fragments were ligated into phCMV2 digested with $B g l \mathrm{II}$ and NotI and its constructs were named HA-NF1-A1/ phCMV2. The expression plasmid for HA-tag fused rat RGPR-p117, HA-RGPR-p117/phCMV2, was constructed as described previously (18).

Western blot analysis. NRK52E cells $\left(1 \times 10^{5}\right)$ were cultured for $72 \mathrm{~h}$ in a medium containing BS. Cells with subconfluency were transiently transfected with HA-NF1-A1/phCMV2 and cultured for $48 \mathrm{~h}$ in DMEM with or without $5 \% \mathrm{BS}$. After culture, the cells were washed three times with phosphatebuffered saline (PBS), scraped into $0.2 \mathrm{ml}$ of ice-cold $0.25 \mathrm{M}$ sucrose solution, and disrupted for $30 \mathrm{sec}$ with an ultrasonic device. Scraped cells were centrifuged at $500 \mathrm{x}$ g for $5 \mathrm{~min}$ to remove cell debris. The $500 \mathrm{~g}$ supernatant was centrifuged at $1,000 \mathrm{x} \mathrm{g}$ for $10 \mathrm{~min}$ to precipitate the nuclear fraction. The $1,000 \mathrm{~g}$ supernatant was used as a cytosolic fraction. The $1,000 \mathrm{~g}$ precipitate was suspended in $0.25 \mathrm{M}$ sucrose and pooled to analyze HA-NF1-A1 by Western blotting. DNA concentration in the $1,000 \mathrm{~g}$ precipitated fraction was determined using the method of Ceriotti (23). The protein concentration in the nuclei and the supernatant fractions was measured using the method of Lowry et al (24).

Aliquots of protein $(20 \mu \mathrm{g})$ were mixed with $5 \mathrm{X}$ Laemmli sample buffer, boiled for $5 \mathrm{~min}$, and SDS-PAGE was performed by the method of Laemmli using $12.5 \%$ polyacrylamide resolving gel (25). After SDS-PAGE, the proteins were then transferred onto polyvinylidene difluoride

A.
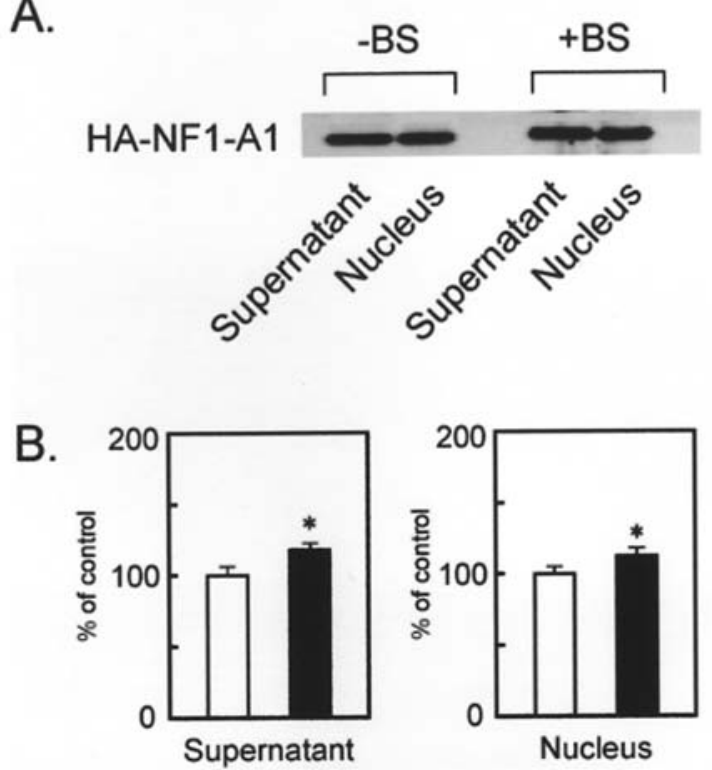

Figure 1. Western blot analysis of HA-NF1-A1 in the nucleus of the cloned normal rat kidney proximal tubular NRK52E cells transfected with HANF1-A1/phCMV2. NRK52E cells $\left(1 \times 10^{5}\right.$ cells) were cultured for $72 \mathrm{~h}$ in a medium containing 5\% BS. Cells with subconfluency were transiently transfected with HA-NF1-A1/phCMV2. The transfectants were cultured for $48 \mathrm{~h}$ in medium with or without $5 \%$ BS. (A) Western blot analysis was carried out on the cell lysate (20 $\mu \mathrm{g}$ protein) obtained from the HA-NF1A1/phCMV2-transfected cells. The figure shows one of four experiments with separate samples. (B) Densitometric data for HA-NF1-A1 levels in transfectants were $121.1 \pm 2.4$ or $115.5 \pm 5.5$ in the cytosol or nucleus (\% of control without BS; mean \pm SEM for four experiments), respectively. These increases were significant $(\mathrm{P}<0.05)$. White bars, control (none); black bars, with BS. 


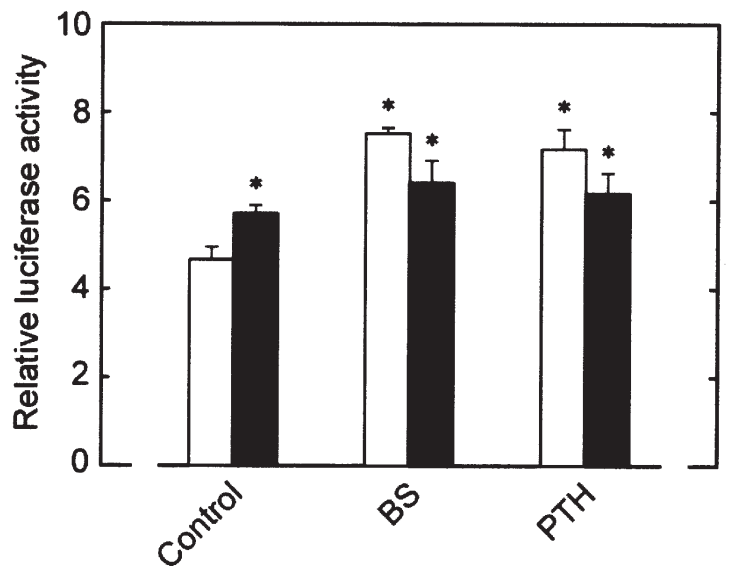

Figure 2. Effects of BS or PTH on promoter activities from the regucalcin promoter $(-718 /+18)$ reporter gene plasmid in the cloned normal rat kidney proximal tubular NRK52E cells (wild-type) or NRK52E cells transfected with HA-NF1-A1/phCMV2 (transfectants). Cells with subconfluency were transiently transfected with HA-NF1-A1/phCMV2. Wild-type cells or transfectants were cultured for $48 \mathrm{~h}$ in medium containing either vehicle, BS $(5 \%)$ or PTH $\left(10^{-7} \mathrm{M}\right)$. Luciferase activity of the test plasmids was corrected for Renilla luciferase activity of the pRL-TK plasmid. The results are expressed as a fold-stimulation in comparison with the luciferase activity measured after transfection with basic LUC, which was set as 1.0. Each value is the mean \pm SEM for the $\%$ of six separate experiments with different cell cultures. ${ }^{*} \mathrm{P}<0.01$ compared with the control value obtained from wild-type cells. White bars, wild-type cells; black bars, HA-NF1A1/phCMV2-transfected cells.

membranes at $100 \mathrm{~mA}$ for $4 \mathrm{~h}$. The membranes were incubated with anti-HA-tag antibody which was diluted 1:1000 in $10 \mathrm{mM}$ Tris- $\mathrm{HCl}$, pH 8.0, containing $150 \mathrm{mM} \mathrm{NaCl}, 0.1 \%$ (w/v) Tween 20 (washing buffer), and 5\% skim milk for $1 \mathrm{~h}$. The membranes were incubated for $1 \mathrm{~h}$ with horseradish peroxidase linked anti-mouse IgG which was diluted 1:2000 with washing buffer containing 5\% skim milk, and again they were washed. Detection of the protein bands was performed using an enhanced chemiluminescent kit following the manufacturer's instructions. The molecular size of the detected protein was determined by running the standard protein molecules of known size in parallel.

Transfection. The cloned normal rat kidney proximal tubular epithelial NRK52E cells (wild-type) were cultured for $72 \mathrm{~h}$ in a medium containing 5\% BS (22). For the transfection experiments, the cells were grown on a 24 -well plate with approximately $70 \%$ confluency and washed once with serumfree DMEM. Either $300 \mathrm{ng}$ of pGL3-Basic plasmid or an equivalent molar amount of test plasmid was co-transfected into NRK52E cells along with $300 \mathrm{ng}$ of pRL-TK plasmid using the synthetic cationic lipid component, a lipofectamin reagent, according to the manufacturer's instructions (Invitrogen). The pRL-TK vector containing the Renilla luciferase gene under control of the herpes simplex virus thymidine kinase promoter was used as an internal control for differences in transfection efficiency and cell number.

After transfection with reporter plasmids, cells were transfected with $300 \mathrm{ng}$ HA-NF1-A1/phCMV2 and/or an equivalent molar amount of HA-RGPR-p117/phCMV2 using lipofectamine (20). For analysis of the regulation of the

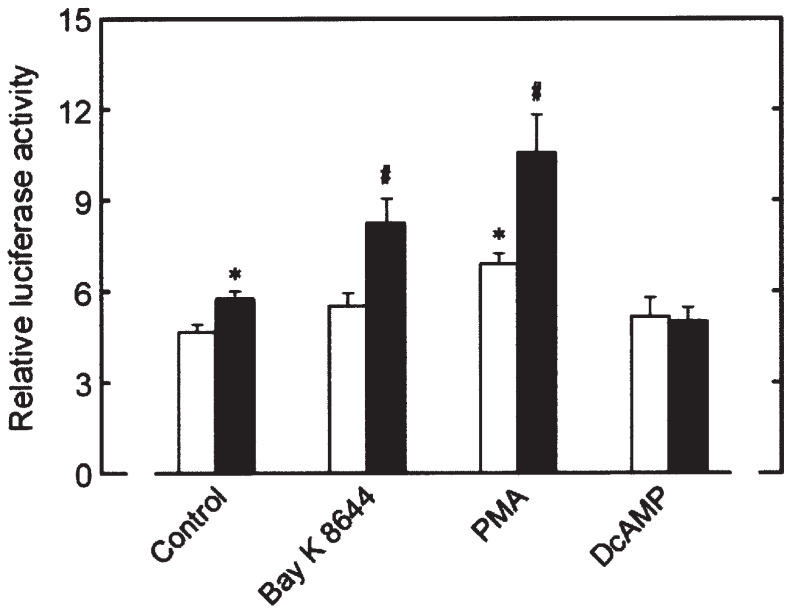

Figure 3. Effects of various intracellular signaling-related factors on promoter activities from the regucalcin promoter $(-710 /+18)$ reporter gene plasmid in the cloned normal rat kidney proximal tubular epithelial NRK52E cells (wild-type) or NRK52E cells transfected with HA-NF1A1/phCMV2 (transfectants). Cells with subconfluency were transiently transfected with HA-NF1-A1/phCMV2 (transfectants). Wild-type cells or transfectants were cultured for $48 \mathrm{~h}$ in medium containing either vehicle, Bay K $8644\left(10^{-6} \mathrm{M}\right)$, PMA $\left(10^{-6} \mathrm{M}\right)$ or DcAMP $\left(10^{-4} \mathrm{M}\right)$. Luciferase activity in the cell lysate was measured as described in the legend of Fig. 2. Each value is the mean \pm SEM for \% of six separate experiments with different cell cultures. ${ }^{*} \mathrm{P}<0.01$ compared with the control value obtained from wild-type cells. ${ }^{~} \mathrm{P}<0.01$ compared with the value without Bay K 8644 or PMA. White bars, wild-type cells; black bars, HA-NF1-A1/phCMV2transfected cells.

regucalcin promoter by signaling factors, the transfected cells were maintained for $48 \mathrm{~h}$ in serum-free DMEM containing either vehicle, $5 \%$ BS, PTH, or various factors. After culture, the transfectants were lysed, and the luciferase activity in the cell lysates was measured using a dual-luciferase reporter assay system (Promega).

Statistical analysis. Data are expressed as the mean \pm SEM. Statistical differences were analyzed using Student's t-test. Pvalues $<0.05$ were considered to indicate statistically significant differences. Also, we used an ANOVA multiple comparison to compare the treatment groups.

\section{Results}

Enhancement of regucalcin gene promoter activity in NRK52E cells transfected with HA-NF1-A1/phCMV2. The cloned normal rat kidney proximal tubular NRK52E cells were cultured for $72 \mathrm{~h}$ in a medium containing $5 \%$ BS. Cells with subconfluency were transiently transfected with HANF1-A1/phCMV2. The transfectants were cultured for $48 \mathrm{~h}$ in medium with or without $5 \% \mathrm{BS}$. The results of the Western blot analysis showed that HA-NF1-A1 was localized in the nuclei of cells (Fig. 1). Culture with BS caused a significant increase in the supernatant and nucleus.

The change in promoter activities from the regucalcin promoter $(-710 /+18)$ reporter gene plasmid in NRK52E cells (wild-type) or NRK52E cells transfected with HA-NF1-A1/ phCMV2 was examined (Fig. 2). Luciferase activity was significantly increased in NRK52E cells transfected with HA-NF1-A1/phCMV2 as compared to that of wild-type 


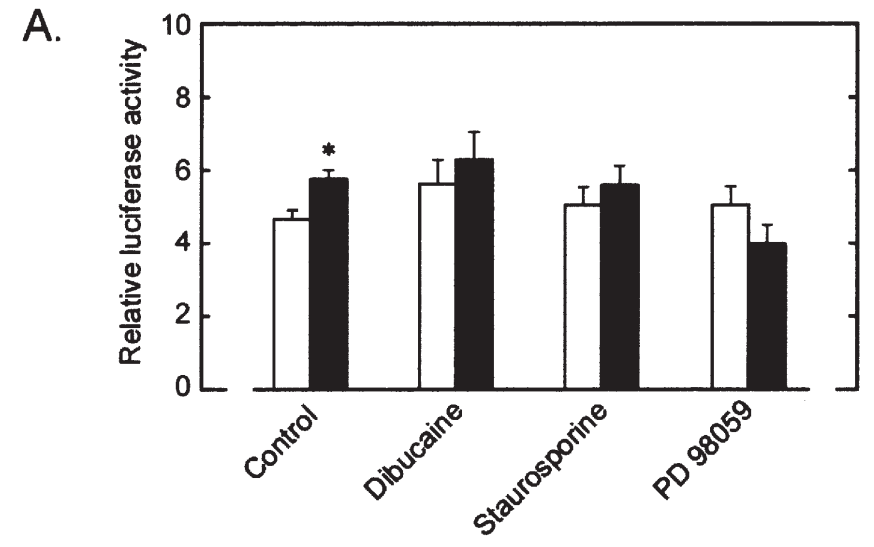

B.

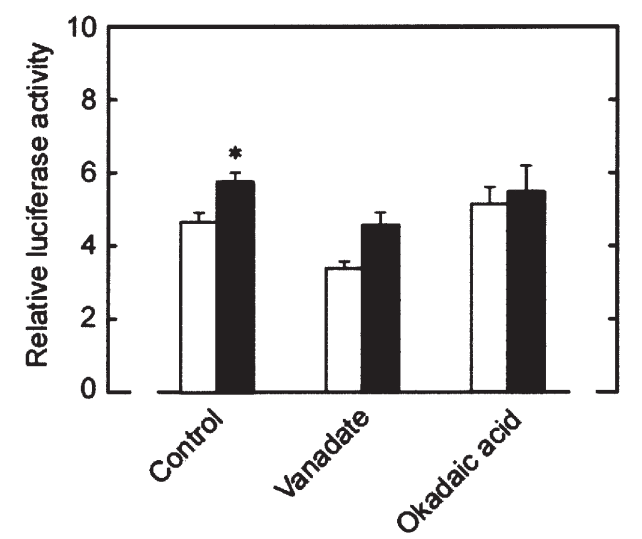

Figure 4. Effects of various intracellular signaling inhibitors on promoter activities from regucalcin promoter $(-710 /+18)$ reporter gene plasmid in the cloned normal rat kidney proximal tubular epithelial NRK52E cells (wild-type) or NRK52E cells transfected with HA-NF1-A1/phCMV2 (transfectants). Cells with subconfluency were transiently transfected with HA-NF1-A1/phCMV2 (transfectants). Wild-type cells or transfectants were cultured for $48 \mathrm{~h}$ in a medium containing either vehicle, dibucaine $\left(10^{-6} \mathrm{M}\right)$, staurosporine $\left(10^{-9} \mathrm{M}\right)$, PD $98059\left(10^{-8} \mathrm{M}\right)$, vanadate $\left(10^{-6} \mathrm{M}\right)$, or okadaic acid $\left(10^{-6} \mathrm{M}\right)$, as shown in A or B. Luciferase activity in the cell lysate was measured as described in the legends of Fig. 2. Each value is the mean \pm SEM for the $\%$ of six separate experiments with different cell cultures. ${ }^{*} \mathrm{P}<0.01$ compared with the control value obtained from wild-type cells. White bars, wild-type cells; black bars, HA-NF1-A1/phCMV2-transfected cells.

cells. The enzyme activity in wild-type cells transfected with the empty vector was not significantly changed as compared to that of wild-type cells (data not shown). The culture with BS $(5 \%)$ or PTH $\left(10^{-7} \mathrm{M}\right)$ caused a significant increase in luciferase activity in wild-type cells compared to that of the untreated group. The increase in luciferase activity in NRK52E cells transfected with HA-NF1-A1/phCMV2 was not significantly enhanced in the presence of BS or PTH compared to that of the untreated group.

Involvement of intracellular signaling factors in the regucalcin gene promoter activity in NRK52E cells transfected with HA-NF1-A1/phCMV2. The effects of intracellular signaling-related factors on promoter activities from the regucalcin promoter $(-710 /+18)$ reporter gene plasmid in NRK52E cells (wild-type) or NRK52E cells transfected with HA-NF1-A1/phCMV2 are shown in Fig. 3. Luciferase activity in wild-type cells was significantly increased in the presence of PMA $\left(10^{-6} \mathrm{M}\right)$ as compared with that of the

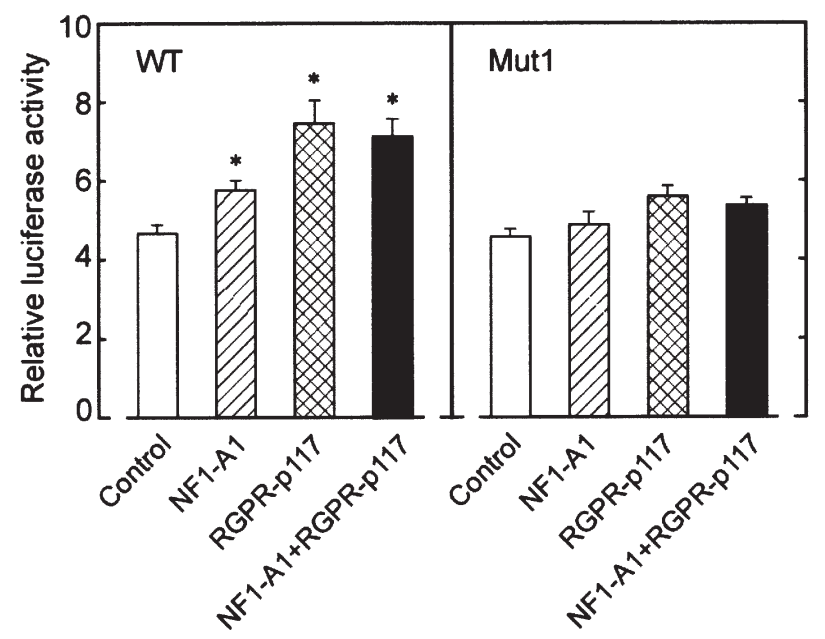

Figure 5. Basal expression of wild-type (WT) and mutant derivative (Mut 1) of the rat regucalcin promoter $(-710 /+18)$ reporter gene plasmid in the cloned normal rat kidney proximal tubular epithelial NRK52E cells (wildtype) or NRK52E cells transfected with HA-NF1-A1/phCMV2, HA-RGPRp117/phCMV2, or HA-NF1-A1/phCMV2 plus HA-RGPR-p117/phCMV2. Cells with subconfluency were transiently transfected with HA-NF1A1/phCMV2, HA-RGPR-p117/phCMV2, or HA-NF1-A1/phCMV2 plus HA-RGPR-p117/phCMV2. Wild-type cells or transfectants were cultured for $48 \mathrm{~h}$ in medium without BS. Luciferase activity in the cell lysate was measured as described in the legends of Fig. 2. Each value is the mean \pm SEM for the \% of six separate experiments with different cell cultures. ${ }^{*} \mathrm{P}<0.01$ compared with the control value obtained from wild-type cells.

control (none), while it was not significantly changed by culture with Bay K $8644\left(10^{-6} \mathrm{M}\right)$ or DcAMP $\left(10^{-4} \mathrm{M}\right)$. Luciferase activity in NRK52E cells transfected with HANF1-A1/phCMV2 was significantly enhanced in the presence of Bay K $8644\left(10^{-6} \mathrm{M}\right)$ or PMA $\left(10^{-6} \mathrm{M}\right)$, while it was not significantly enhanced by culture with DcAMP $\left(10^{-4} \mathrm{M}\right)$.

The effects of intracellular signaling inhibitors on promoter activities from the regucalcin promoter reporter gene plasmid in NRK52E cells (wild-type) or NRK52E cells transfected with HA-NF1-A1/phCMV2 are shown in Fig. 4. The effect of HA-NF1-A1/phCMV2 transfection in increasing luciferase activity was not seen in the presence of dibucaine $\left(10^{-6} \mathrm{M}\right)$, an antagonist of calmodulin (26), staurosporine $\left(10^{-9} \mathrm{M}\right)$, an inhibitor of protein kinase C (27), or PD $98059\left(10^{-8} \mathrm{M}\right)$, an inhibitor of MAP kinase (28), with an effective concentration (Fig. 4A). Such an effect was also observed in the presence of vanadate $\left(10^{-6} \mathrm{M}\right)$ or okadaic acid $\left(10^{-6} \mathrm{M}\right)$, an inhibitor of protein phosphatase (29) (Fig. 4B).

Involvement of the TTGGC motif in the regucalcin gene promoter in NRK52E cells transfected with HA-NF1-Al/ phCMV2 or HA-RGPR-p117/phCMV2. Whether the TTGGC motif in the rat regucalcin promoter region is involved in the enhancement of regucalcin mRNA expression in NRK52E cells overexpressing NF1-A1 was examined. Wild-type cells or transfectants with subconfluency were transfected with either $-710 /+18$ LUC construct (WT) or chimeric construct containing serial deletion [-523/-435 (mutant 1 )] of the regucalcin promoter region that includes the TTGGC motif (20). The increase in luciferase activity in NRK52E cells transfected with HA-NF1-A1/phCMV2 was not seen in mutant 1 (Mut 1) (Fig. 5). 


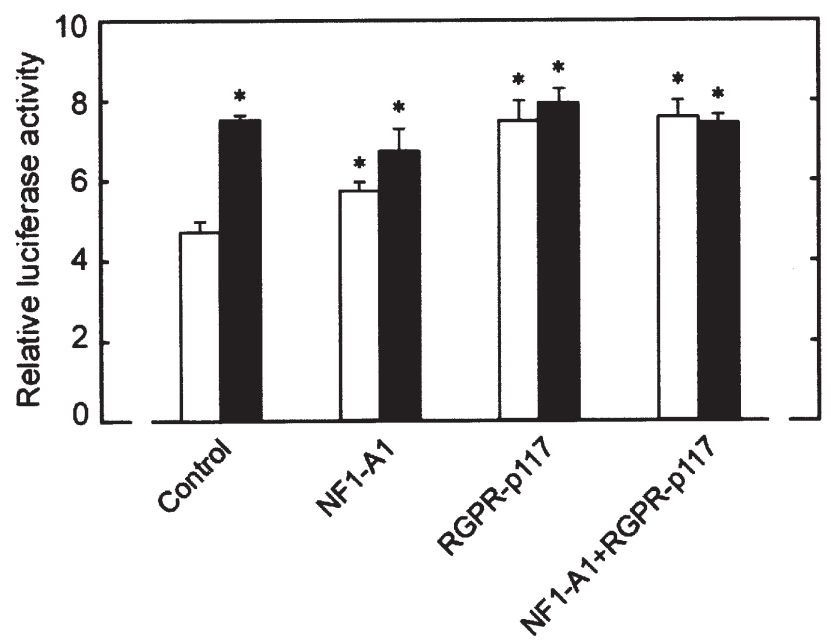

Figure 6. Effects of BS on promoter activities from the rat regucalcin promoter $(-710 /+18)$ plasmid in the cloned normal rat kidney proximal tubular epithelial NRK52E cells transfected with HA-NF1-A1/phCMV2 and/or HA-RGPR-p117/phCMV2. Cells with subconfluency were transiently transfected with HA-NF1-A1/phCMV2 and/or HA-RGPRp117/phCMV2. Wild-type cells or transfectants were cultured for $48 \mathrm{~h}$ in medium containing either vehicle or BS $(5 \%)$. Luciferase activity in the cell lysate was measured as described in the legends of Fig. 2. Each value is the mean \pm SEM for the $\%$ of six separate experiments with different cell cultures. ${ }^{*} \mathrm{P}<0.01$ compared with the control (none) value obtained from wild-type cells (WT). White bars, without BS; black bars, with BS.

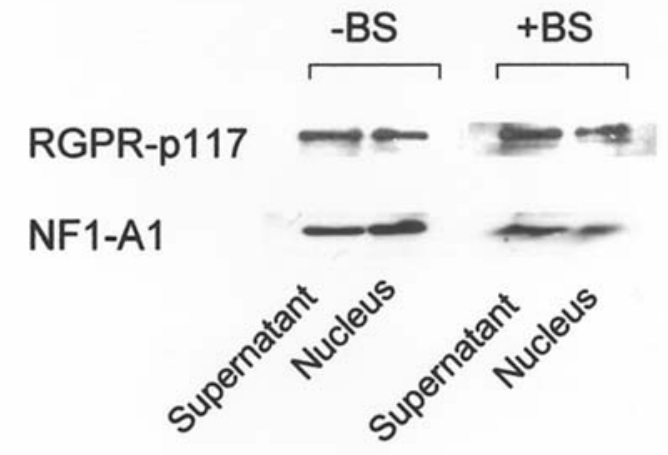

Figure 7. Western blot analysis of NF1-A1 and HA-RGPR-p117 in the nucleus of the cloned normal rat kidney proximal tubular epithelial cells transiently transfected with HA-NF1-A1/phCMV2 and HA-RGPR-p117/ phCMV2. Cells with subconfluency were transiently transfected with HANF1-A1/phCMV2 and HA-RGPR-p117/phCMV2. Wild-type cells or transfectants were cultured for $48 \mathrm{~h}$ in medium with or without $5 \% \mathrm{BS}$ Western blot analysis was carried out on the cell lysate $(20 \mu \mathrm{g}$ protein) obtained from transfectants. The figure shows one of four experiments with separate samples.

Overexpression of RGPR-p117 can enhance the rat regucalcin promoter activity that is involved in the TTGGC motif in the rat regucalcin promoter region (20). Luciferase activity was significantly increased in NRK52E cells transiently transfected with HA-RGPR-p117/phCMV2 (Fig. 5). This increase was not seen in mutant 1 (Mut 1). In addition, the increase in luciferase activity in NRK52E cells transfected with HA-NF1-A1/phCMV2 was not significantly enhanced in the cells transiently transfected with HA-RGPRp117/ phCMV2 (Fig. 5).

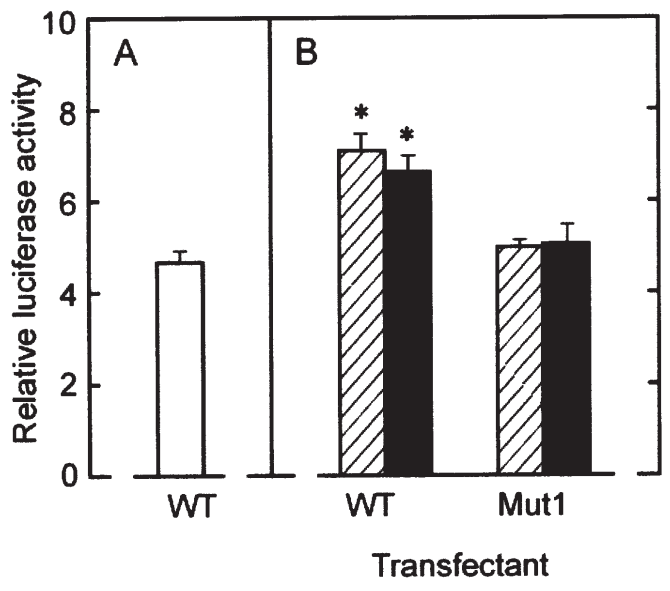

Figure 8. Effect of NF1-A1 expression in promoter activities from rat regucalcin promoter $(-710 /+18)$ with wild-type cells (WT) and mutant derivatives (Mut 1) in the cloned normal rat kidney proximal tubular NRK52E cells (A; wild-type) or NRK52E cells overexpressing HA-RGPRp117 stably (B). HA-RGPR-p117-overexpressing cells with subconfluency were transiently transfected with HA-NF1-A1/phCMV2, and the cells were cultured for $48 \mathrm{~h}$ in medium without BS. Luciferase activity in the cell lysate was measured as described in the legend of Fig. 2. Each value is the mean \pm SEM for the $\%$ of six separate experiments with different cell cultures. ${ }^{*} \mathrm{P}<0.01$ compared with the control value obtained from wild-type cells. White bar, control (wild-type); hatched bars, stably HA-RGPR-p117overexpressed cells; black bars, stably HA-RGPR-overexpressed cells transiently transfected with HA-NF1-A1/phCMV2.

Effect of $B S$ on the regucalcin gene promoter activity in NRK52E cells transfected with HA-NF1-A1/phCMV2. The effects of $\mathrm{BS}$ on promoter activities from the regucalcin promoter $(-710 /+18)$ reporter plasmid in NRK52E cells (wild-type) or NRK52E cells transiently transfected with HA-NF1-A1/phCMV2, HA-RGPR-p117/ phCMV2, or HANF1-A1/phCMV2 plus HA-RGPR-p117/phCMV2 were examined (Fig. 6). Culture with BS (5\%) caused a significant increase in luciferase activity in wild-type cells. The presence of BS $(5 \%)$ did not significantly enhance the increase in luciferase activity in NRK52E cells transfected with HANF1-A1/phCMV2, HA-RGPR-p117/phCMV2, or HA-NF1A1/phCMV2 plus HA-RGPR-p117/phCMV2.

The results of Western blot analysis showed that NF1-A1 or RGPR-p117 was localized in the nucleus of NRK52E cells transiently transfected with HA-NF1-A1/phCMV2 and HARGPR-p117/phCMV2 (Fig. 7). NF1-A1 or RGPR-p117 was co-localized in the nucleus of cells transfected with HA-NF1A1/phCMV2 plus HA-RGPR-p117/phCMV2 (data not shown).

Effect of NF1-A1 expression on the regucalcin gene promoter activity in NRK52E cells overexpressing RGPR-p117 stably. The effect of NF1-A1 expression on promoter activities from rat regucalcin promoter $(-710 /+18)$ with wild-type (WT) and mutant derivative (Mut 1) in the cloned normal rat kidney proximal tubular NRK52E cells (wild-type) or NRK52E cells overexpressing HA-RGPR-p117 stably was examined (Fig. 8). Overexpression of RGPR-p117 caused a significant increase in luciferase activity in the wild-type (WT) cells, as shown previously (Sawada and Yamaguchi, 20). This increase was 
not significantly enhanced in the RGPR-p117-overexpressed NRK52E cells (wild-type) transiently transfected with HANF1-A1/phCMV2. The increase in luciferase activity in the RGPR-p117-overexpressed NRK52E cells (wild-type) or the RGPR-p117-overexpressed cells transiently transfected with HA-NF1-A1/phCMV2 was not seen in the mutant 1 .

\section{Discussion}

NF1-A1 and RGPR-p117 are identified as a hepatic nuclear factor that binds to the TTGGC sequence of the rat regucalcin gene promoter region using a yeast one-hybrid system $(15,16)$. Overexpression of RGPR-p117 has been shown to enhance the rat regucalcin gene promoter activity that is involved in the TTGGC sequence in cloned normal rat kidney proximal tubular epithelial NRK52E cells $(19,20)$. This study demonstrates that NF1-A1 increases the rat regucalcin gene promoter activity in NRK52E cells, and that this enhancement is not revealed in the mutant with deletion of the TTGGC(N) ${ }_{6} \mathrm{CC}$ sequence in the cells.

NF1-A1 was found to localize in the nuclei of NRK52E cells transiently transfected with HA-NF1-A1/phCMV2, and it enhanced the rat regucalcin gene promoter activity in the cells. This increase was not significantly enhanced by culture with BS or PTH, while the culture with BS or PTH caused a significant increase in the rat regucalcin gene promoter activity in NRK52E cells (wild-type). However, culture with Bay K 8644 or PMA significantly enhanced the regucalcin gene promoter activity in NRK52E cells transiently transfected with HA-NF1-A1/phCMV2. Bay K 8644 is an agonist of calcium entry into cells (30). PMA is an activator of protein kinase $\mathrm{C}(31)$. It is speculated that $\mathrm{Ca}^{2+}$-dependent protein kinases are partly involved in the enhancement of the regucalcin gene promoter activity in NRK52E cells transiently transected with HA-NF1-A1/phCMV2.

The increase in the regucalcin gene promoter activity in NRK52E cells transiently transfected with HA-NF1-A1/ phCMV2 was completely blocked by culture with dibucaine, staurosporine, PD 98059, vanadate, or okadaic acid. The result suggests that $\mathrm{Ca}^{2+}$-dependent protein kinases, MAP kinase, and protein phosphatases are involved in the enhancement of regucalcin gene promoter activity in NRK52E cells transfected with HA-NF1-A1/phCMV2. Presumably, various intracellular signaling factors are involved in the enhancement of the regucalcin gene promoter activity in NRK52E cells transfected with HA-NF1-A1/phCMV2.

RGPR-p117 increases the rat regucalcin gene promoter activity in NRK52E cells transfected with the -710/+18 LUC construct vector (20). Co-transfection with HA-NF1-A1 and HA-RGPR-p117/phCMV2 did not significantly enhance the RGPR-increased regucalcin gene promoter activity in NRK52E cells transfected with the $-710 /+18$ LUC construct vector or the $-710 /+18$ LUC construct with deletion of $-523 /-435$. RGPR-p117 or NF1-A1 was localized in the nuclei of transfectants. NF1-A1 or RGPR-p117 may regulate independently in the enhancement of the rat regucalcin gene promoter activity in NRK52E cells. When HA-NF1-A1/ phCMV2 was transiently transfected into NRK52E cells overexpressing RGPR-p117 stably, the regucalcin gene promoter activity in the RGPR-p117-overexpressed cells was not significantly changed. This observation may support the view that the regucalcin gene promoter activity is independently regulated by NF1-A1 or RGPR-p117 that binds to the TTGGC motif in the promoter region.

NF1-A1 and RGPR-p117 were identified as a hepatic nuclear factor that binds to the TTGGC $(\mathrm{N})_{6} \mathrm{CC}$ sequence of the rat regucalcin gene promoter region using a yeast onehybrid system $(15,16)$. NF1-A1 or RGPR-p117 could increase the rat regucalcin gene promoter activity in NRK52E cells. This increase was involved in the TTGGC $(\mathrm{N})_{6}$ sequence in the promoter region of the rat regucalcin gene. Various signaling factors may be involved in the regulation of the rat regucalcin gene promoter activity in NRK52E cells overexpressing NF1-A1 or RGPR-p117. This enhancement seems to be mediated through protein phosphorylation and dephosphorylation in NRK52E cells. NF1-A1 or RGPR-p117, however, regulates independently in the enhancement of the rat regucalcin gene promoter activity in NRK52E cells. In addition, RGPR-p117 is a novel protein $(15,17)$, and it works as a transcription factor (20). NF1-A1 and RGPR-p117 may play a role as a transcription factor in the rat regucalcin promoter activity.

In conclusion, it has been demonstrated that NF1-A1 increases the rat regucalcin gene promoter activity in cloned normal rat kidney proximal tubular NRK52E cells.

\section{Acknowledgements}

This work was supported in part by a Grant-in aid for Scientific Research (C), no. 17590063 from the Ministry of Education, Science, Sports, and Culture, Japan.

\section{References}

1. Yamaguchi $\mathrm{M}$ and Yamamoto T: Purification of calcium binding substance from soluble fraction of normal rat liver. Chem Pharm Bull 26: 1915-1918, 1978.

2. Shimokawa $\mathrm{N}$ and Yamaguchi $\mathrm{M}$ : Molecular cloning and sequencing of the cDNA coding for a calcium-binding protein regucalcin from rat liver. FEBS Lett 327: 251-255, 1993.

3. Yamaguchi $\mathrm{M}$ and Mori S: Effect of $\mathrm{Ca}^{2+}$ and $\mathrm{Zn}^{2+}$ on $5^{-}-$ nucleotidase activity in rat liver plasma membranes: hepatic calcium-binding protein (regucalcin) reverses the $\mathrm{Ca}^{2+}$ effect. Chem Pharm Bull 36: 321-325, 1988.

4. Yamaguchi $\mathrm{M}$ : A novel $\mathrm{Ca}^{2+}$-binding protein regucalcin and calcium inhibition. Regulatory role in liver cell function. In: Calcium Inhibition. Kohama K (ed). Japan Sci Soc Press, Tokyo and CRC Press, Boca Raton, pp19-41, 1992.

5. Yamaguchi M: Role of regucalcin in calcium signaling. Life Sci 66: 1769-1780, 2000.

6. Yamaguchi M: The role of regucalcin in nuclear regulation of regenerating liver. Biochem Biophys Res Commun 276: 1-6, 2000 .

7. Yamaguchi M: Role of regucalcin in maintaining cell homeostasis and function (Review). Int J Mol Med 15: 371-389, 2005.

8. Misawa $\mathrm{H}$ and Yamaguchi M: The gene of $\mathrm{Ca}^{2+}$-binding protein regucalcin is highly conserved in vertebrate species. Int J Mol Med 6: 191-196, 2000.

9. Yamaguchi M, Makino R and Shimokawa N: The 5' end sequences and exon organization in rat regucalcin gene. Mol Cell Biochem 165: 145-150, 1996.

10. Murata $\mathrm{T}$ and Yamaguchi M: $\mathrm{Ca}^{2+}$ administration stimulates the binding of AP-1 factor to its 5'-flanking region of rat gene for the $\mathrm{Ca}^{2+}$-binding protein regucalcin. Biochem J 329: 157-163, 1998.

11. Murata $\mathrm{T}$ and Yamaguchi M: Promoter characterization of the rat gene for $\mathrm{Ca}^{2+}$-binding protein regucalcin. Transcriptional regulation by signaling factors. J Biol Chem 274: 1277-1285, 1999. 
12. $\mathrm{Xu} \mathrm{M}$, Osada $\mathrm{S}$, Imagawa $\mathrm{M}$ and Nishihara $\mathrm{T}$ : Genomic organization of the rat nuclear factor I-A gene. J Biochem 122 : 795-801, 1997.

13. Misawa $\mathrm{H}$ and Yamaguchi $\mathrm{M}$ : Involvement of hepatic nuclear factor I binding motif in transcriptional regulation of $\mathrm{Ca}^{2+}$ binding protein regucalcin gene. Biochem Biophys Res Commun 269: 270-278, 2000.

14. Misawa $\mathrm{H}$ and Yamaguchi $\mathrm{M}$ : Intracellular signaling factorsenhanced hepatic nuclear protein binding to TTGGC sequence in the rat regucalcin gene promoter: Involvement of protein phosphorylation. Biochem Biophys Res Commun 279: 275-281, 2000.

15. Misawa $H$ and Yamaguchi $M$ : Molecular cloning and sequencing of cDNA coding for a novel regucalcin promoter region-related protein in rat, mouse, and human liver. Int $\mathrm{J} \mathrm{Mol}$ Med 8: 513-520, 2001.

16. Misawa $\mathrm{H}$ and Yamaguchi $\mathrm{M}$ : Identification of transcription factor in promoter region of rat regucalcin gene: binding of nuclear factor I-A1 to TTGGC motif. J Cell Biochem 84: 795-802, 2002.

17. Sawada $\mathrm{N}$ and Yamaguchi $\mathrm{M}$ : A novel regucalcin promoter region-related protein: Comparison of nucleotide and amino acid sequences in vertebrate species. Int J Mol Med 15: 97-104, 2005.

18. Sawada N, Nakagawa T, Murata T and Yamaguchi M: Nuclear localization of novel protein RGPR-p117 in normal rat kidney proximal tubular epithelial cells. Int J Mol Med 16: 809-814, 2005.

19. Sawada N and Yamaguchi M: Overexpression of RGPR-p117 enhances regucalcin gene expression in cloned normal rat kidney proximal tubular epithelial cells. Int J Mol Med 16: 1049-1055, 2005.

20. Sawada N and Yamaguchi M: Overexpression of RGPR-p117 enhances the regucalcin gene promoter activity in cloned normal rat kidney proximal tubular epithelial cells: Involvement of TTGGC motif. J Cell Biochem (In press).
21. Rice EK, Tesck GH, Cao Z, Cooper ME, Metz CN, Bucala R, Atkins RC and Nikilic-Peterson DJ: Induction of MIF synthesis and secretion by tubular epithelial cells: a novel action of angiotensin II. Kidney Int 63: 1265-1275, 2003.

22. Nakagawa T and Yamaguchi M: Hormonal regulation on regucalcin mRNA expression in cloned normal rat kidney proximal tubular epithelial NRK52E cells. J Cell Biochem 95: 589-597, 2005.

23. Ceriotti G: Determination of nucleic acids in animal tissues. $\mathbf{J}$ Biol Chem 241: 59-70, 1955.

24. Lowry OH, Rosebrough NH, Farr AL and Randall RF: Protein measurement with the Folin phenol reagent. J Biol Chem 193: 265-273, 1951.

25. Laemmli UK: Cleavage of structural proteins during the assembly of the head of bacteriophage T4. Nature 224: 680-685, 1970.

26. Vincenzi FE: Pharmacology of calmodulin antagonism. In: Calcium Modulators. Godfraind T, Albertini A and Paoletti R (eds). Elservier Biomedical Press, Amsterdam, pp67-80, 1982

27. Tamaoki T, Nomoto H, Takahashi I, Kato Y, Morimoto M and Tomita F: Staurosporine, a potent inhibitor of phospholipid/ $\mathrm{Ca}^{2+}$ dependent protein kinase. Biochem Biophys Res Commun 135: 397-402, 1986.

28. Pelech SL, Charest DL, Mordret GP, Siow YL, Palaty C, Campbell D, Charlton L, Samiei M and Sanghera JS: Networking with mitogen-activated protein kinases. Mol Cell Biochem 127: 157-169, 1993.

29. Hunter T: Protein kinases and phosphatases: The yin and yang of protein phosphorylation and signaling. Cell 80: 225-236, 1995.

30. Cano-Abad MF, Villarroya M, Garcia AG, Gabilan NH and Lopez MG: Calcium entry through L-type calcium channels causes mitochondrial disruption and chromaffin cell death. J Biol Chem 276: 39695-39704, 2001.

31. Nishizuka Y: Studies and perspectives of protein kinase C. Science 233: 305-312, 1986. 